\title{
Ética Profesional en la Universidad: Dilemas y tensiones en la formación de un sujeto ético-político
}

\author{
Professional Ethics at the University: Dilemmas and tensions in the development of an ethical- \\ political individual \\ Douglas A. Izarra Vielma ${ }^{a}$, Cecilia Navia $^{b}$
}

\begin{abstract}
:
Teaching professional ethics is part of the everyday actions and interactions of formal and informal institutions. The objective of this paper is to discuss the teaching process of professional ethics at the university; for that purpose, we propose some dilemmas, problems and questions to foster the debate. We base on two important assumptions: a) every educational action has a moral dimension and $b$ ) the teaching of ethics must be directed to the development of an ethical-political individual. We highlight the important of assuming this task with responsibility, choosing relevant contents, developing different and original strategies, and avoiding the routinization of teaching practices; above all, teachers must reflect on the different consequences of creating ethicalpolitical subjects, who exercise their profession for the benefit of society.
\end{abstract}

\section{Keywords:}

Professional ethics, university, formation.

\begin{abstract}
Resumen:
La enseñanza de la ética profesional ocurre en las acciones e interacciones educativas de la vida cotidiana de una institución, tanto formales como informales. En tal sentido se establece como objetivo discutir sobre la formación de la ética profesional en la universidad, con este propósito se plantean dilemas y tensiones y se formulan preguntas que intentan promover el debate. Dos supuestos resultan centrales en esta discusión: a) toda acción educativa tiene una dimensión moral y, b) la enseñanza de la ética debe orientarse a la formación de un sujeto ético-político. Se resalta la importancia de asumir responsablemente esta tarea, seleccionando contenidos pertinentes, desarrollando estrategias diversas y originales, evitando la rutinización de las prácticas, pero sobre todo, reflexionado acerca de las implicaciones de formar sujetos ético-políticos que ejerzan su profesión en beneficio de la sociedad.
\end{abstract}

\section{Palabras Clave:}

Ética profesional, universidad, formación

\section{Introducción}

\section{¿De qué hablamos cuando hablamos de enseñar ética profesional?}

La preparación integral de los profesionales en la universidad debe incluir, de acuerdo con Bolívar (2005), por lo menos tres componentes: conocimientos especializados, habilidades técnicas y marco de conducta en la actuación laboral. En relación con la tercera cuestión es que se formula el objetivo del presente ensayo: discutir sobre el proceso de formación en ética profesional en la universidad. Para ello, se hace necesaria la identificación de dilemas y tensiones que se presentan y la formulación de interrogantes que permitan analizar el tema.

Como punto de partida asumimos dos supuestos: a) toda acción educativa tiene una dimensión moral y, b) la enseñanza de la ética debe orientarse a la formación de un sujeto ético-político. Sobre el primer supuesto, de acuerdo con Cullen (2009: 79), "entendemos por dimensión moral de los problemas educativos todo aquello que en la acción de educar puede ser materia o sujeto de un razonamiento ético-práctico". Tal definición 
permite asegurar que las prácticas educativas de una universidad tienen implicaciones morales que se evidencian en la toma de decisiones y en la relación con los otros.

El segundo supuesto lleva a plantear la pregunta ¿a qué nos referimos cuando hablamos de enseñar ética profesional?, que puede favorecer a la configuración del ethos profesional, entendido como "sistema disposicional que se activa frente a problemas del ámbito socio-moral en el campo profesional" (Yurén, 2013:8).

La enseñanza de la ética profesional puede desarrollarse en la universidad desde dos opciones: su inclusión como contenido del plan de estudios o como un elemento que promueve el desarrollo de procesos reflexivos sobre problemas y dilemas que se presentan en la práctica. Si se apunta al primero, nos referimos al ámbito del currículo, bien como una materia específica, contenidos o actividades vinculadas con el ejercicio de una profesión.

Desde la intención de desarrollar procesos reflexivos en los estudiantes, la ética profesional debe atravesar todas las prácticas que se dan en la universidad, incluyendo a la institución como organización y como programa institucional (Dubet, 2006). Esto incluye los diferentes espacios o instancias que al interior de la misma existen $y$, toca también al currículo, en su dimensión formal (cursos o contenidos, ejes transversales) o informal (en las acciones e interacciones cotidianas de los actores, vinculado con el currículo oculto).

Consideramos que es desde esta segunda posición, que integra a la universidad en la complejidad de sus espacios y actores, que es posible vincular claramente los dos supuestos que nos formulamos al inicio, a partir de dos cuestiones: el lugar de la ética profesional se encuentra en toda la universidad (considerando que toda acción educativa, e incluso de gestión, siempre tiene implicaciones morales) y que, la universidad tiene la responsabilidad de incidir en la preparación ético-política de sus estudiantes y de todos sus actores (docentes, personal administrativo y de servicio), desde un proceso reflexivo que les permita partir de principios y razones para validar o legitimar sus acciones.

De acuerdo con lo anterior, son múltiples los escen arios que ofrece la universidad para el desarrollo de la formación de un sujeto ético-político, que "tiene la actitu d y la facultad para convocar, liderar y participar en el debate público [con el propósito de] transformar las estructuras sociales que atentan contra la justicia, la dignidad y el buen vivir de todos y todas" (Rivera, 2014: 169). No se trata solo de contribuir a la formación moral del estudiante, sino que consideramos que se hace cada vez más necesaria, para la construcción de una sociedad justa, que posibilite el pleno desarrollo de todos sus ciudadanos, una formación ética-política.

\section{Ética profesional}

\section{¿Por qué formar un sujeto ético-político?}

Parte importante de las discusiones y aportes a la ética profesional provienen del campo de la bioética y del campo médico, aunque también, desde el área de las comunicaciones, la industria de la guerra y los problemas del cambio climático. Entre las declaraciones e instrumentos que han permitido reflexionar en torno a las implicaciones éticas del ejercicio de una profesión, destacan el Código de Nuremberg (1947), la Declaración de Helsinki (1964), el Informe Belmont (1979) y, más recientemente, la Declaración Universal sobre Bioética y Derechos Humanos de la UNESCO (2005), ligada a la Declaración Universal de Derechos Humanos (1948), entre otras.

Aun cuando se reconoce la importancia de estos y otros acuerdos sobre el tema, es necesario cuestionar las posibilidades que tienen para influir en la regulación e impacto en las prácticas de los profesionales en la universidad, su poco alcance para la atención de los problemas de las minorías de género, grupos indígenas, migrantes, grupos etáreos, entre otros, cuyos derechos no se encuentran del todo, o de manera clara, in cluidos en estos acuerdos.

No cabe duda que son antecedentes que permiten abrir la discusión en torno a la ética profesional, pero no son suficientes. De ahí que, siguiendo a Yurén (2005:28), en el contexto actual "prevalece una lógica homogeneizante e instrumental a la que hay que oponer resistencias que se concretan en procesos de individuación y subjetivación". En este escenario cobran valor las iniciativas que permiten el desarrollo de personas conscientes de las circunstancias y capaces de asumir una actitud crítica ten diente al cambio social.

Si bien las universidades despliegan dispositivos de formación para el mundo del trabajo, en la práctica su papel puede ser cuestionado. Rivera (2014: 171) se pregunta al respecto: "¿qué responsabilidad tiene la academia en la formación de sujetos ético-políticos? Es más, ¿la academia se vende al poder de las instituciones o se compromete con la formación de la sociedad civil?". De acuerdo con el autor, es posible que estas instituciones no se beneficien con el logro de ciudadanos críticos y agentes de cambio.

De ahí que es necesario cuestionar los procesos de formación en las universidades cuando estos se cen tran en el desarrollo de competencias laborales, desde una perspectiva técnica o instrumental, pero con una eticidad deficiente, pues ello conduce al desarrollo de prácticas que terminan por reproducir estructuras sociales profundamente injustas.

Frente a estos cuestionamientos, las instituciones de educación superior tienen la responsabilidad de 
reconocer las relaciones de fuerza que se dan en su interior, en las que se hacen presentes, desde diferentes actores, dilemas y tensiones en la formación profesional y política de los futuros profesionales.

De acuerdo con Bolívar (2005: 94), "plantearse lo que deba ser la formación de un profesional competente no es posible al margen de una formación ética y una educación para la ciudadanía". Esta idea implica que desarrollar estudios en la universidad, más allá del logro de competencias, debe necesariamente apuntar al desarrollo de un sólido compromiso que permita asumir el ejercicio de la profesión como un espacio que trasciende lo exclusivamente laboral.

En este sentido, Beauchamp y Childress (2001), exponen que las profesiones consisten en actuaciones que tienen como propósito realizar un determinado tipo de bienes o servicios. A partir de ello, plantean como principios éticos fundamentales para la ética profesional el respeto por las personas, su dignidad y autonomía, la beneficencia, la no maleficencia y la justicia.

Coincidiendo con lo anterior, Adela Cortina explica que desde su origen las profesiones están concebidas como una actividad que "trasciende a quien la sirve" (Cortina, 2006: 58), en tanto están encaminadas a favorecer la colectividad. Similar idea se encuentra en el trabajo de Hortal (2002: 52), quien señala que es inherente al ejercicio profesional prestar un servicio a la sociedad para el cual se debe desarrollar un "proceso de capacitación teórica y práctica".

Articulado con la formación del profesional en su condición de actor social, de la relación con los otros y con el mundo en el cual está inserto, es importante articular la formación del sujeto ético-político, considerando sus posibilidades de acción en relación con su proyecto personal, al cuidado de sí y al desarrollo de su autonomía. Esto implica, contribuir a preparar sujetos capaces de actuar en relación a sí mismos y a los otros, basados en lo que aprenden a lo largo de la vida. En palabras de Yurén (2013: 13) "la configuración de una personalidad capaz de criticar los valores existentes, colocarse en el punto de vista moral, juzgar con prudencia las situaciones con miras a la equidad y, sobre todo, actuar reflexiva y autocríticamente".

Desarrollar la preparación en torno a una actuación ética en el ejercicio de la profesión, supone ir más allá de plantear acciones concretas para aplicar en la práctica. En ese escenario son innumerables y en muchos casos, imprevisibles los problemas éticos que se pueden presentar. Más que pensar la ética profesional desde el pensamiento especulativo por el bien o lo bueno, se trata de plantear cómo utilizar el pensamiento crítico en la resolución de los problemas específicos a los que nos enfrentamos cotidianamente en el mundo laboral y en el día a día de las instituciones de educación superior.
Como profesionales, es importante reconocer, y tomar en cuenta, que los profesionales se enfrentan a contextos particulares, en los que la actividad laboral se realiza mediada por la interacción social. A través de ésta se configuran un conjunto de representaciones, relativamente estables, compartidas por los profesionales e internalizadas, a través del proceso de instrucción y socialización, que orientan las prácticas de orden socio moral en un campo laboral. De ahí que la ética configura una cultura profesional, ante la cual se establecen formas de apropiación e identitarias (Yurén, 2003).

Según Hortal (2002), existen tres mediatizaciones fundamentales a las que se enfrentan las profesiones: Ia mediatización técnica, la mediatización económica y la mediatización organizativa o institucional. La primera hace referencia a las consecuencias éticas que implica ampliar las capacidades profesionales, pues si bien se puede ser competente técnicamente, ello no implica que necesariamente se sea responsable en el ejercicio profesional. Ser un mero técnico puede restringir el campo laboral, por ejemplo, cuando se invierte la jerarquía entre medios y fines, planteando sólo aqu ellas tareas y metas para las que hay técnicas elaboradas y desarrolladas, o cuando se sobrevalora la mentalidad técnica, por encima de las posibilidades de respuesta y responsabilidades éticas de los sujetos, condu ciendo al profesional a ser un técnico instrumentalizado, a aumentar la alienación y dificultar la construcción de una identidad coherente, configurando una profesionalidad performativa (Luengo y Molina-Pérez, 2019).

Siguiendo con Hortal (2002), la mediatización económica hace referencia al profesional que trabaja para una empresa o para una organización, cuya responsabilidad como experto se encuentra mediada fundamentalmente en términos económicos $u$ organizativos. La mediatización institucional y organizativa se relaciona con los modos habitualizados y tipificados para actuar en las instituciones, que los profesionales internalizan en el proceso de socialización, haciendo suyo el modo de hacer y de ser del grupo con los que comparte labor. De ahí que la responsabilidad en la actividad laboral y las actuaciones éticas de la persona y como profesional, están mediadas por el entorno social y la cultura compartida.

Como seres humanos, siempre nos confrontamos con el hecho ético, pero es fundamental establecer una distancia ética frente a las normas y principios que se nos plantean, a las que se les dota, por la tradición, de un sentido universal (Ricoeur, 2010: 33). Es decir, cuando nos planteamos la actualidad de estas normas y principios, en los casos particulares de su aplicación, se antepone "una reinterpretación en el presente que se apodere de la objetivación y el distanciamiento". Esta distancia ética, se convierte en productiva, en el sentido 
de favorecer la interpretación de posibilidades de una actuación competente. De ahí que podemos señalar que, en la enseñanza de la ética profesional en las universidades, no está desligada de la libertad y las posibilidades de realización de los sujetos en el campo laboral, en tanto, siguiendo nuevamente a Ricoeur (2010; 34) "la vida ética es una transacción perpetua entre el proyecto de la libertad y su situación ética, delineada por el mundo dado de las in stitu ciones".

En concordancia con lo anterior, para Yurén (2013) la formación en ética profesional que se despliega en las universidades debe orientarse hacia la praxis, combinando tres instancias: saber, actuar moral y hacer, que se vinculan con el concepto de agencia el cual resulta coherente con la concepción expuesta del sujeto ético-político.

\section{Ética profesional}

\section{¿Qué espacios ofrece la universidad para la mediación en el desarrollo de un sujeto ético- político?}

Las instituciones de educación superior, como organizaciones complejas, ofrecen múltiples espacios que pueden afectar los procesos de formación universitaria, particularmente en su dimensión éticopolítica. En tal sentido, se consideran tanto el plano organizacional, como el diseño cu rriculary, por supuesto, los espacios de aula tradicionalmente considerados como los más relevantes.

Existe un amplio consenso que reconoce al contexto social como un espacio para el aprendizaje. Así por ejemplo, se encuentran los postulados de Vigotsky (1978) respecto al aprendizaje sociocultural y de Bandura (1987) en relación con el aprendizaje social. Monereo (2007) propone un enfoque sociocognitivo de aprendizaje estratégico que ayuda a identificar los mecanismos sociales y personales que influyen en este proceso. Este último autor reconoce la importancia de la interacción del estudiante con la cultura institucional en la que desarrolla sus estudios como parte in dispensable de lo que denomina "conocimiento estratégico".

Por otro lado, Yurén (2005), recuperando a Habermas, menciona la existencia de plexos de sentido social entendidos como órdenes normativos en los que han sido socializados los sujetos.

Desde el reconocimiento de la importancia del contexto, como espacio para el aprendizaje, se puede asu mir que la universidad, como organización, con un programa institucional, puede favorecer o también entorpecer la mediación para la preparación ético-política de sus miembros.

Lo anterior tiene profundas implicaciones. Considerando que son múltiples las vías para la formación, es posible plantear, como Celia y Vera (2016) sugieren, "la indiferencia también enseña". Los autores señalan que cuando en las instituciones se realizan acciones que pueden ser consideradas injustas o contrarias a la norma, pueden constituirse en un modelo negativo, pues quienes se forman pueden percibir que el criterio que orienta la toma de decisiones no privilegia lo académico. En sentido contrario, y de acuerdo con Cullen (2009), Ia enseñanza de la ética se facilita en presencia de contextos institucionales democráticos, regidos por políticas educativas justas. Este proceso no incluye únicamente a los estudiantes, es extensible también a los profesores y a todo el personal de las universidades e instituciones de educación superior.

La circunstancia descrita entraña cierta dificultad, pues en el contexto universitario siempre prevalecen tensiones en torno al tipo de institución que se pretende construir; a qué tipo de sujetos se pretende formar, así como, a las relaciones que se espera establecer con la sociedad. Esto incide, por ejemplo, en la forma en que se toman las decisiones de funcionamiento y las de tipo curricular, y cómo éstas son percibidas, negociadas y puestas en acción por la comunidad académica.

Puede resultar obvio que las universidades, de acuerdo con la misión que socialmente se les atribuye, se preocupen por la preparación en ética profesional de sus estudiantes y profesores, tanto en lo relacionado con el ejercicio de la profesión, como con la práctica de la investigación. En tal sentido se aprecia de forma cada vez más frecuente la creación de instancias para tratar el tema. Un ejemplo de ello, es la creación de comités de ética, en los cuales han tenido una amplia influencia los aportes y discusiones que se han desarrollado a nivel internacional, tales como los mencionadas en la sección anterior de este trabajo.

Especial importancia ha tenido la discusión sobre ética de la investigación en las universidades. De acuerdo con Fuentes y Revilla (2007), se crean comités con la finalidad de proteger el bienestar y seguridad de los participantes de una investigación, evaluar el balance riesgo/ben eficio de un estudio y valorar su rigor científico y la factibilidad. Sin embargo, en la práctica, estas instancias sufren la tensión entre el deber ser para el que fueron creadas y constituirse en espacios para la reflexión o el reducirse a la ejecución de prácticas rutinarias que pueden terminar por convertir su dinámica en una cuestión de carácter eminentemente bu rocrática o normativa. De hecho, Fuentes y Revilla señalan como un problema para estos órganos que su trabajo no es valorado por los propios investigadores, al considerarlo como una carga bu rocrática o una instancia de control. Los comités de ética de la investigación pueden ver reducido su funcionamiento al diseñar formularios para la presentación adecuada de proyectos, verificar requisitos 
que deben cumplir los proyectos presentados por estudiantes y profesores de la universidad, ofrecer información en relación con la legislación vigente y finalmente, emitir resultados de la evaluación de los protocolos de investigación y, con ello, se identifica que contribuyen poco con la instrucción en ética de la investigación de los universitarios, puesto que puede conducir a asociar la ética con una cuestión eminentemente burocrática y, en el peor de los casos, punitiva.

En las universidades también se incorpora la ética profesional a partir del diseño cu rricular. De acuerdo con Hirsch (2014), esto se puede hacer a través de diversas vías: como una materia o curso, como eje transversal que permea el currículo o como un contenido asociado con su puesta en práctica. Para Arteaga (2005: 249), "la transversalidad constituye una forma de permear los saberes", y según Molina (2007), surgió como estrategia para abordar la diversidad y de esa manera lograr la formación integral del individuo. Comúnmente se acepta que los ejes transversales permiten el tratamiento, en la universidad, de cuestiones relevantes para el alumno y que además, favorecen el acercamiento de los estudios universitarios a la vida cotidiana.

Sin embargo, la incorporación de la ética profesional, como un tema transversal del currículo universitario, no está exenta de problemas, tales como la cuestión de ¿cómo permea la práctica del trabajo docente? Es probable que no todos los profesores comprendan las implicaciones de la transversalidad en su práctica pedagógica, y que solo represente un aspecto significativo para algunos que estén realmente interesados en la formación ética de sus estudiantes. También es posible que como tema transversal se convierta en un conjunto de actividades extracurriculares, sin conexión directa con la dinámica de lo que ocu rre en las aulas y menos aún, con los problemas a los que los estudiantes se pueden enfrentar en su vida en el mu ndo del trabajo.

\section{Enseñanza de la ética profesional}

\section{¿Aprobar o formar sujetos ético-políticos?}

Además de la incorporación de la ética profesional como tema transversal, y de incluirla en el currículo a través de contenidos en el contexto de diversas asignaturas (normalmente vinculadas con el ejercicio laboral) y a través de cursos específicos (Hirsch, 2014), como señalamos más arriba, incorporarla en el currículo permite su tratamiento de forma directa en el trabajo del profesor universitario, y garantiza que en los planes de estudio se consideren espacios y tiempos para la instrucción en esta área.

La vinculación de la ética con el ejercicio profesional tiene importantes beneficios, por ejemplo, en la reflexión de la cotidianeidad del trabajo en situaciones reales y el consiguiente desarrollo de la sensibilidad sobre las implicaciones de la profesión en el contexto social en el cual se va a ejercer. Sin embargo, para su logro es indispensable que la formación se articule con el resto del currículo y contar con una actitud adecuada por parte de docentes y estudiantes.

La incorporación de los contenidos y cursos de ética debe responder a las finalidades del currículo como un todo y ser parte de un proceso general que se desarrolle en las universidades, de lo contrario estaría sign ado por contradicciones que limitarían sus beneficios. En este sentido, De Vicente et al. (citados por Bolívar, 2005) realizaron una investigación en la Universidad de Granada, España, en la que obtuvieron como resultado que la atención prestada a la enseñanza de principios éticos en la preparación del magisterio es escasa. En un contexto diferente, en el estado Táchira, en Venezuela, Izarra (2016) consultó a un grupo de docentes en relación con las experiencias significativas que les permitieron formarse en valores profesionales durante sus estudios universitarios. Las respuestas que se obtuvieron a esta interrogante fueron múltiples, vinculadas a la relación con los compañeros de trabajo, a la práctica de la enseñanza, al ejercicio en in stituciones educativas, entre otras. Sin embargo, cuando los entrevistados en el estudio, se refirieron al desarrollo o a la participación en los cursos que están previstos sobre ética profesional en los programas de formación docente, no consideraron su formación en valores profesionales como una experiencia significativa. Estos estudios revelan la importancia de considerar el trabajo que pueden realizar los profesores que administran tales asignaturas.

Es importante reflexionar sobre la actitud con la que docentes y estudiantes asumen los procesos de enseñanza y aprendizaje de la ética profesional. Si los cursos son percibidos únicamente como un requisito que es necesario aprobar, los estudiantes los pueden asumir como una carga académica, que únicamente tiene como fin de obtener la calificación necesaria y pasar el curso. Se encuentra así, una tensión entre la necesidad de aprobar el curso y formar sujetos ético-políticos capaces de saber, pensar y actuar en función de los alcances de su profesión para el espacio social, en el cual van a desarrollarla. Este aspecto se analizará de manera específica, desde la didáctica, en el siguiente apartado.

\section{Didáctica de la ética profesional}

$$
\text { ¿Cuestión de contenidos y estrategias? }
$$


De acuerdo con lo expuesto, la ética profesional en la universidad permea diferentes espacios institucionales, curriculares y deontológicos. Específicamente, en el plano del currículo se encuentra la opción de incorporarla como tema transversal, contenido o curso específico. Desde estas opciones (particularmente las dos últimas) resulta especialmente relevante considerar el tema desde el papel de la didáctica.

Hemos mencionado que al incorporar cursos o seminarios relacionados con la ética profesional, se corre el riesgo de que sea asumida por el estudiante como una obligación académica, una materia más que es necesario aprobar para alcanzar la titulación. Igualmente señalamos que para evitar esta situación resulta relevante el desempeño del profesor y particularmente, los recursos y estrategias que utilice para desarrollar el proceso de enseñanza en el contexto de unos contenidos previstos en el diseño curricular respectivo.

Al partir del desarrollo de contenidos previamente establecidos en un programa, el profesor es responsable de encontrar un equilibrio, asumiendo la presencia constante de puntos de tensión en la formación de los profesionales y la trasposición didáctica del conjunto de contenidos que están más allá de su elección. En algunas oportunidades los cursos pueden estar circunscritos a la deontología, haciendo que los contenidos se presenten como un conjunto de códigos y postulados de obligatorio cumplimiento, asumiendo un principio de enseñanza prescriptivo. También es posible que los contenidos se refieran a postulados teóricos, muchas veces reducidos a definiciones conceptuales sobre qué es ética, moral, ética profesional, ética de la investigación, valores, entre otros conceptos. En ese caso, la enseñanza se centra en una dimensión cognitiva, abstracta, que dificulte la posibilidad de comprender los posibles usos prácticos de la misma. En otros casos, la preparación ética puede presentarse como algo neutral, desligado de los problemas políticos, sociales y económicos, que constituyen el marco desde el cual es posible el ejercicio en el ámbito laboral.

Es el profesor, a partir de su experticia, de su posición y visión de la universidad y la sociedad, quien tiene la responsabilidad de usar los contenidos para promover la formación de sus estudiantes como sujetos éticopolíticos. Para ello precisa, desde su capacidad de agencia (enfrentándose a situaciones dilemáticas), poner en juego la trasposición didáctica. Pongamos como ejemplo los enunciados de los códigos deontológicos. Estos pueden ser analizados a la luz de ejemplos extraídos en la práctica o de los problemas a los que se puede enfrentar el docente en los contextos organizacionales, institucionales y sociales donde ejerce la profesión.
Desde este enfoque didáctico se lograría la vinculación entre postulados teóricos y el ejercicio profesional (en tre lo abstracto del principio y lo concreto de la actividad laboral). De igual manera, se puede orientar a los estudiantes para que a partir de principios generales, encuentren casos de aplicación particular (por ejemplo, diferenciar en la práctica ética y moral desde sus propias definiciones).

Lo anterior revela la importancia del rol docente en el proceso de formación ético-política de los estudiantes y lleva a las preguntas: ¿qué competencias didácticas se requieren para enseñar ética?, ¿se puede enseñar tal como se enseñan otras disciplinas, como geografía, historia, biología o matemática? Consideramos que además de tener un dominio en los conocimientos disciplinares, es importante que el profesor comprenda la distinción de la ética profesional, como campo disciplinar y como campo de prácticas, reconociendo a su vez, que su enseñanza trasciende el dominio de unos contenidos disciplinares específicos, y que requiere una contextualización de las prácticas. Por esa razón, se hace necesario promover investigaciones didácticas en el campo de la enseñanza de la ética profesional (Cullen, 2009), así como indagar desde el uso de diversos procedimientos metodológicos.

López (2014) presenta múltiples estrategias que pu eden contribuir a la formación del estudiante, entre las que destacan: el uso de dilemas morales, el juego de roles, la escritura autobiográfica, frases inacabadas y preguntas clarificadoras, la resolución de conflictos y actividades de comprensión crítica, entre otras. Sin embargo, más allá de la diversidad de técnicas, siempre debe estar presente una intención formativa en cada actividad que se espera desarrollar, vinculada a la intención de formar un tipo de persona o profesional. Esto supone asumir que el profesor, como agente educativo, parte de un conjunto de principios que entran en juego con la intencionalidad del curriculum, las condiciones socioculturales del contexto y las mediatizaciones en el ejercio de la profesión.

De acuerdo con Bolívar, el profesor requiere asumir la responsabilidad respecto a que:

...la enseñanza de la ética tiene como objetivo proporcionar instrumentos y claves relevantes para tener criterios propios y capacidad de elección propios de ciudadanos que participan en los asuntos públicos (2005: 96).

De ahí la insistencia en asumir que la formación ética se orienta, finalmente, al desarrollo de la capacidad de agencia y al compromiso político, que involucra a la vez, tanto al profesor como al estudiante. 


\section{En conclusión}

\section{¿Cuál es el lugar para la formación en ética profesional?}

Discutir con relación al lugar de la ética profesional en la universidad nos remite a reflexionar sobre la necesidad de formar personas para el cambio social. Esto supone romper con la lógica instrumental que puede estar orientando la acción de las universidades y entorpecer el logro de sus propósitos fundamentales.

Se hace necesario, cada vez más, cuestionar cuáles son las intenciones o los grandes propósitos que debemos considerar cuando queremos formar a nuestros estudiantes. Se trata de transmitir contenidos, desarrollar códigos, crear comités, o más bien estamos frente a la necesidad de formar profesionales críticos, que se asuman como sujetos ético-políticos, que siguen principios de justicia y autonomía, que buscan producir beneficios para la sociedad, más que contribuir a su deterioro. Nos referimos a los principios de la ética profesional, mencionados anteriomente, como son: justicia, autonomía, beneficencia y no-maleficencia ni maledicencia.

La universidad no puede sustraerse de ellos si quiere mantenerse en un proceso de reflexión constante de sus finalidades y del lugar que ocupa en la sociedad y en la formación profesional. Por esa razón, se trata de ir más allá de contenidos y estrategias; se pretende retomar la importancia de un proceso reflexivo permanente de parte de las instituciones de educación superior y de sus actores en la formación de personas dispuestas a contribuir efectivamente con el cambio de la sociedad, más allá de cumplir con una actividad laboral.

El desarrollo de profesionales, como sujetos éticopolíticos en la universidad, se presenta como un imperativo para contrarrestar el simple eficientismo que pretende circunscribir el trabajo a un conjunto de competencias estandarizadas. También impele a la universidad cuestionar la lógica neoliberal e instrumental en sus procesos formativos, que favorecen el desarrollo de prácticas profesionales que no toman en cuenta las desigualdades e injusticias presentes en la sociedad. Lejos de ello, se plantea la formación de sujetos éticopoliticos, capaces de actuar, desde un cuestionamiento ético constante (con un sentido dilemático), para asu mir una práctica profesional basada en principios éticos, sentido crítico y responsable.

\section{Referencias}

Arteaga, Marlene (2005). Modelo tridimensional de transversalidad. Investigación y Postgrado, 20 (2) pp. 241-274. Recuperado de http://www.redalyc.org/articulo.oa $? \mathrm{id}=65820209$

Asociación Médica Mundial(1964). Declaración de Helsinki. Recuperado de https://www.um.es/documents/7232477/7272232/declaracion_de_helsi nki.pdf/643a 79f0-980d-4a95-a442-5fb1 dee 81548

Bandura, Albert (1987). Pensamiento y Acción: fundamentos sociales. Barcelona (España): Martínez Roca

Beauchamp, Tom y Childress, James (2001). Principles of Biomedical Ethics. New York: Oxford University Press.

Bolívar, Antonio (2005). El lugar de la ética profesional en la formación universitaria. Revista Mexicana de Investigación Educativa, 10 (24), pp. 93-123. Recuperado de http://comie.org.mx/documentos/rmie/v10/n24/pdf/rmiev 10n24scB06n 01 es.pdf

Celia, Adriana y Vera, Gumersindo (2016). La Indiferencia También Enseña. Ponencia presentada en el II Congreso de la Red Iberoamericana de Investigadores en Ética y Responsabilidad Social Universitaria. Instituto de Investigaciones sobre la Universidad y la Educación (IISUE) de la Universidad Nacional Autónoma de México

Comisión Nacional para la Protección de los Sujetos Humanos de Investigación Biomédica y del Comportamiento (1979). Informe Belmont. Recuperdo de http://www.bioeticayderecho.ub.edu/archivos/norm/InformeBelmont.p df

Cortina, Adela (2006). Universalizar la aristocracia: por una ética de las profesiones. Claves de razón práctica, Revista Cultural de Santander, 1, $54 \quad$ - 65.6 Recuperado de http://revistas.uis.edu.co/index.php/revista santander/article/view/2186/ 2547

Cullen, Carlos (2009). Entrañas Éticas de la Identidad Docente. Buenos Aires (Argentina): La Crujía

Dubet, François (2006), El declive de la institución. Profesiones, sujetos e individuos ante la Reforma del Estado, Barcelona: Gedisa.

Fuentes, Duilio y Revilla, Diana (2007). Acreditación de comités de ética en investigación como parte de un proceso. Anales de la Facultad de

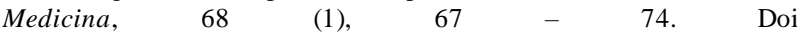
https://doi.org/10.15381/anales.v68i1.1241

Hirsch, Ana (2014). Formación en ética profesional para estudiantes universitarios. En A. Hirsch y R. López (Coords.), Ética Profesional en Educación Superior: Finalidades, estrategias y desafíos de la formación.(pp. 39 - 58). Sinaloa: Universidad Autónoma

Hortal, Augusto (2002). Ética General de las Profesiones. Bilbao: Desclée De Brower

Izarra, Douglas (2016). Experiencias para la formación en valores de docentes estudiantes de postgrado. En D. Izarra Vielma (Coord.), Experiencias y perspectivas en ética profesional y responsabilidad social universitaria en Iberoamérica, pp. 13-29. Recuperado de http://ciegc.org.ve/2015/wp-content/uploads/2016/05/\%C3\% 89tica-yRSU.pdf

López, Mery (2014). Construyendo Ciudadanos: educación, ciudadanía y convivencia en Venezuela. Mérida: Universidad de Los Andes Consejo de Publicaciones

Luengo, Julián y Molina-Pérez, Javier (2019). Construyendo la resistencia profesional en un espacio educativo neoliberalizado. Educatio Siglo XXI, 37 (1) 57 - 75. Doi https://doi.org/10.15366/reice2020.18.2.003

Molina, Denyz (2007). Ejes transversales en el currículo universitario: experiencia en la carrera de derecho. Ciências \& Cognição, 10, 132$147 . \quad$ Recuperado de: http://pepsic.bvsalud.org/scielo.php?script=sci_arttext\&pid=S180658212007000100013\&lng=pt\&tlng=pt.

Monereo, Carles (2007). Hacia un nuevo paradigma del aprendizaje estratégico: el papel de la medicación social, del self y de las emociones. Revista Electrónica de Investigación Psicoeducativa, 5 (3) pp. 497-534. Recuperado de http://www.investigacionpsicopedagogica.org/revista/articulos/13/espannol/Art_13_206.pdf 
Ricoeur, Paul (2010). Ética y cultura. Buenos Aires: Prometeo Libros / Universidad Católica Argentina.

Rivera, Álvaro (2014). Escenarios para la formación del sujeto éticopolítico en América Latina. Revista Educación y Desarrollo Social, 8 (1), pp. 162-179. Recuperado de: https://revista s.unimilitar.edu.co/index.php/reds/article/view/564

Tribunal Internacional de Núremberg (1947). Código de Núremberg. Recuperado http://www.bioeticanet.info/documentos/Nuremberg.pdf

UNESCO, (2005). Declaración Universal sobre Bioética y Derechos Humanos. Recuperado de: https://unesdoc.unesco.org/ark:/48223/pf0000142825_spa.page=85

Vygotsky, Lev (1978). El Desarrollo de los Procesos Psicológicos Superiores. Barcelona (España): Grijalbo.

Yurén, M. Teresa (2003). Tensiones identitarias y ethos profesional. El caso del profesor de formación cívica y ética en la escuela secundaria. En A. Hirsch y R. López (coords.) Ética profesional e identidad institucional, pp. 267-295. Culiacán, México, Universidad Autónoma de Sinaloa.

Yurén, M. Teresa (2005). Ethos y autoformación en los dispositivos de formación de docentes. En M. Yurén, C. Navia y C. Saenger (Coords.) Ethos y Autoformación del Docente. pp. 19-45. Barcelona (España): Ediciones Pomares

Yurén, M. Teresa (2013). Ética profesional y praxis. Una revisión desde el concepto de "agencia". Perfiles Educativos, XXXV (142) pp. 6-14. Recuperado de: https://www.iisue.unam.mx/perfiles/articulo/2013142-\%C3\%89tica-profesional-en-la-educacion-superior.pdf 